\title{
Leitthema
}

Hautarzt 2013 - 64:748-751

DOI 10.1007/s00105-013-2593-2

Online publiziert: 21. Oktober 2013

๑) Springer-Verlag Berlin Heidelberg 2013
K. Fritz ${ }^{1,2,3} \cdot$ G.S. Tiplica ${ }^{2} \cdot$ C. Salavastru ${ }^{2} \cdot$ M. Onder ${ }^{1,4}$

${ }^{1}$ Haut und Laser Centrum - Landau

2 Universität Carol Davila, Bucharest

${ }^{3}$ Universitäten Bern und Osnabrück, Bern

${ }^{4}$ Gazi Universität Ankara Medizinische Fakultät , Ankara

\begin{abstract}
Alitretinoin (9-cis-Retinsäure) ist ein Vertreter der Retinoide, die in vivo als Vitamin und als Hormon agieren. Die Wirkung beruht auf Interaktionen mit beiden intrazellulären Retinoid-Rezeptorentypen [allen ,Retinoic acid receptors“" (RAR)- und „Retinoid X receptors" (RXR)-Subtypen] im Unterschied zu anderen Retinoiden. Die aktivierten Rezeptoren agieren als Transkriptionsfaktoren, die die Expression von Genen regulieren, was wiederum Zelldifferenzierung und Proliferation bei gesunden und neoplastischen Zellen steuert. Alitretinoin hat dabei einen ausgeprägteren antiinflammatorischen Effekt als andere Retinoide.
\end{abstract}

Alitretinoin ist zugelassen für die Behandlung des schweren chronischen Handekzems, das nicht oder nicht ausreichend auf die Behandlung mit potenten topischen Kortikosteroiden anspricht. Es ist ein Agonist beider Vitamin-A-Säure-Rezeptoren und wirkt primär immunmodulatorisch und antientzündlich. Schon bei diesem Wirkungsspektrum und der Nähe zu anderen Vitamin-A-Derivaten ergeben sich potenzielle Einsatzmöglichkeiten für andere auf Vitamin A ansprechende Dermatosen. Alitretinoin wird daher bereits „off label“ auch bei anderen chronisch entzündlichen und dermatoonkologischen Hauterkrankungen wie mediterranem Kaposi-Sarkom, kutanen TZell-Lymphomen, Psoriasis und starker Akne eingesetzt. Die perorale Gabe bei epithelialen Tumoren wird in Studien geprüft. Seit April 2005 ist Alitretinoin auch als Gel zur Lokaltherapie für Patienten mit Aids-bedingtem Kaposi-Sarkom in Deutschland verfügbar. Das bereits 2000 von der EMEA zugelassene Präparat ist angezeigt zur topischen Behandlung von Hautläsionen, wenn eine Radio- oder Chemotherapie nicht indiziert ist und die Knoten frei von Ulzera und Lymphödem sind und nicht auf eine systemische antiretrovirale Therapie ansprechen.

Im Folgenden soll ein Überblick gegeben werden, bei welchen nicht zugelassenen Indikationen Alitretinoin bislang eingesetzt und in Publikationen, Falldarstellungen oder Kongressbeiträgen darüber berichtet wurde sowie mit welchem Ergebnis.

\section{Vitamin-A-sensible Hauterkrankungen}

Psoriasis und verwandte Erkrankungen, Genodermatosen mit Störungen der Keratinisation, Akne und Photoaging sind klassische und anerkannte Indikationen für Retinoide, weiterhin epitheliale Präkanzerosen und Tumore, Seborrhö, Rosazea, akneiforme Dermatosen, Lichen planus, eosinophile Follikulitis, Condylomata accuminata, Lichen sclerosus et atrophicus und manche mehr. Bei einigen davon wurde bereits über den Einsatz von Alitretinoin berichtet [41].

\section{Palmoplantare Psoriasis}

Eine palmoplantare Psoriasis, die unter Etanacept und Vitamin D und zuzüglich Methotrexat keine Besserung zeigte und auch zu einem Wiederauftreten nach Absetzen führte, zeigte deutliche Befundbesserung nach 4 Wochen und Abheilung nach 8 Wochen unter der Kombination von Etanercept plus $30 \mathrm{mg} / \mathrm{Tag}$ Alitretinoin [20].

In einem eigenen Fall konnte ein therapieresistentes chronisches psoriasiformes Hand- und Fußekzem bei latent aktivier- barer Poliomyelitis mit $30 \mathrm{mg}$ Alitretinoin erfolgreich behandelt werden [9]. Ebenso besserte sich der Hautbefund bei Patienten mit einer beruflich provozierten Psoriasis palmaris [26].

\section{Palmoplantare pustulöse Psoriasis}

Sieben Patienten erhielten 1-mal täglich 12 Wochen lang $30 \mathrm{mg}$ Alitretinoin.

Der „pustular psoriasis area and severity index“ (PPPASI) und die ,visual analogue scales" (VAS) für die Intensität der Schmerzen bzw. des Pruritus wurden jeweils unter dieser Therapie signifikant um $60-90 \%$ in der Patientenbeurteilung reduziert. In der Histologie war eine deutliche Abnahme von Neutrophilen, Makrophagen und dendritischen Zellen nachweisbar. Dieses Ergebnis wird allerdings durch die Tatsache eingeschränkt, dass es sich um eine Studienbeobachtung ohne Kontrollgruppe handelt und zusätzlich Lokaltherapie zugelassen war [13].

\section{Palmoplantare \\ hyperkeratotische Psoriasis}

Bei einer schweren palmoplantaren hyperkeratotischen Psoriasis konnte ein besseres Ergebnis unter Etanercept und Alitretinoin in Kombination erreicht werden.

Alitretinoin kann also wirksam mit Anti-tumor-Necrosis-Faktor-blockierenden Wirkstoffen kombiniert werden [20].

\section{Morbus Darier}

Verschiedene Publikationen belegen die Wirkung bei Morbus Darier. Ein 18-jähriger Patient erreichte schon nach der ersten Woche mit einer Dosierung von $30 \mathrm{mg}$ täglich Besserung, besonders des Juckreizes und des Erythems. Ab der 4. Woche 
verminderten sich Schuppung und Fissuren, nach 52 Wochen war der Patient bis auf minimale Restherde beschwerdefrei bei sonst guter Verträglichkeit [3].

Auch über jeweils 2 Frauen im gebärfähigen Alter wurde berichtet, die eine fast vollständige Abheilung erreichten, am ehesten wohl durch die antiproliferative und antiinflammatorische Wirkung [40].

\section{Ichthyosis}

In der Behandlung der Ichthyosis variegata (OMIM 609165) bei einem Patienten zeigte sich eine rasche Besserung, bei der lamellaren Ichthyosis erst bei höherer Dosierung bis $50 \mathrm{mg} /$ Tag über 3 Monate [10].

Bei Zellulitis der Kopfhaut bei Keratitis-Ichthyosis-Taubheits-Syndrom wurden deutliche Verbesserungen erzielt [37].

\section{Pityriasis rubra pilaris}

Bei einem therapieresistenten Verlauf trotz Einsatzes fast sämtlicher lokaler und systemischer Medikation kam es bei einer 74-jährigen Patientin unter Alitretinoin nach nur wenigen Wochen zur deutlichen Besserung. Juckreiz und Schuppung verschwanden - ein Ergebnis, das bei therapeutisch schwierigen Fällen zu einem Einsatz von Alitretinoin ermutigt [31, 21].

\section{Morbus Hailey Hailey}

Zwei Fälle des therapeutisch schwierigen Verlaufs eines Morbus Hailey Hailey sind ebenfalls berichtet worden.

Es handelt sich dabei um eine autosomal-dominante, akantholytische Erkrankung, bedingt durch den Verlust der $\mathrm{Mu}$ tation des ATP2C1-Gens, die zu einer Insuffizienz des Golgi-assoziierten $\mathrm{Ca}^{2+}$ Transporters führt [30].

\section{Lichen planus}

Unter 30 mg/Tag konnte auch ein palmoplantarer Lichen planus in Kombination mit Creme-PUVA (3- bis 4-mal/Woche) und topischer Steroid- und Salicylgabe innerhalb von 5 Monaten gebessert werden, wobei die palmaren Veränderungen schneller als die plantaren ansprachen [22].
Bei 3 Patientinnen, davon 2 mit Manifestation an Haut und Mundschleimhaut, besserte sich der Befund bereits nach 4 Wochen unter 30 bzw. $10 \mathrm{mg}$ pro Tag [8].

Der Lichen planus kann in bis zu 10\% auch die Nägel befallen bis hin zur „twenty nail dystrophy“. Unter $30 \mathrm{mg}$ Alitretinoin konnten in 1 Fall nicht nur nach 6 Wochen die Hautbefunde deutlich gebessert werden, sondern auch die Nägel, wobei diese langsam nach distal gesund und unbeeinträchtigt herauswuchsen und somit erst nach 24 Wochen eine vollständige Abheilung zeigten. Die Verträglichkeit war gut [31].

Es wurde auch über einen Lichen planus, der nach Hepatitisimpfung auftrat und erfolgreich mit Alitretinoin behandelt werden konnte, berichtet [33].

Der Einfluss von Retinoiden auf die Nagelbildung ist nicht ganz aufgeklärt, es gibt aber Hinweise auf die Rezeptorexpression an der Nagelmatrix und den Einfluss auf das Nagelwachstum $[27,36]$.

\section{Lupus erythematodes}

Retinoide gehören in der Behandlung des Lupus erythematodes (LE) nach den Antimalariamedikamenten nur zu den Therapien der zweiten Wahl. Für Acitretin wurde eine Wirkung bereits nachgewiesen.

Bei 3 Patienten konnte Alitretinoin eingesetzt werden [1 Patient mit systemischem Lupus erythematodes (SLE), 2 Patienten mit diskoidem Lupus erythematodes (DLE)], nachdem andere Therapeutika versagt hatten. In allen Fällen stellten sich Besserungen ein und führten je danach Ausprägung und Typ zur vollständigen Abheilung nach nur 4 Wochen in 1 Fall, in den anderen nach 2 bzw. 5 Monaten bei einer Dosis von 30 mg/Tag [16].

\section{Atopische Dermatitis}

Vier Männer und 2 Frauen erhielten wegen eines extrapalmaren atopischen Ekzems bei gleichzeitig bestehendem chronischem Handekzem 12 Wochen $30 \mathrm{mg}$ Alitretinoin pro Tag und zusätzlich eine topische Therapie. Darunter verbesserte sich nicht nur das Handekzem, sondern auch die extrapalmaren Läsionen verbesserten sich um mehr 50\% im SCORAD
(Score of Atopic Dermatitis) und PGA (Physician’s Global Assessment; [11]).

\section{Fußekzem}

Nicht nur Handekzeme sind mit Alitretinoin behandelbar, sondern natürlich auch Fußekzeme, auch wenn dies in der Zulassung als Indikation nicht explizit aufgeführt ist [35].

\section{Alopecia areata}

Bei der Behandlung eines chronischen Handekzems mit 30 mg/Tag Alitretinoin wurde eine gleichzeitig bestehende Alopecia areata allmählich besser mit vollständiger Remission nach 11 Monaten. Eigentlich führen Vitamin-A-Derivate als Nebenwirkung zum Haarausfall, die Autoren vermuten allerdings, dass in niedriger Dosis der RXR-Rezeptor Haarwachstum induzieren kann, wie es von Bexeroten bei der systemischen Anwendung beim kutanen T-Zell-Lymphom (CTCL) bekannt ist [15].

\section{Stoffwechselstörungen}

In einem Einzelfall wurde berichtet, dass ein Dorfman-Chanarin-Syndrom mit Alitretinoin verbessert werden kann [38].

\section{Hautfunktion und Hautbarriere}

Unter der Behandlung mit Alitretinoin soll es zu Veränderungen der Hautbarrierefunktion und der Hautlipide kommen und zu erhöhter Irritabilität auf „sodium lauryl sulphate“ (SLS). Untersuchungen an 8 Patienten unter 2-monatiger Alitretinoin-Therapie hat keine Änderung dieser Art bestätigen können, auch nicht im transepidermalen Wasserverlust (TEWL; [14]).

\section{Einsatz bei malignen und prämalignen Veränderungen}

Tretinoin und Tazarotene sind in der Behandlung lichtgeschädigter Haut wirksam, binden aber nur RAR-Subtypen, Alitretinoin in 0,1\%-Gelform (Panretin) hingegen bindet und aktiviert sowohl RARs als auch RXRs. Unter Lokalbehandlung benigner Hautveränderungen wie sebor- 
rhoischer Keratosen als auch von präkanzerösen aktinischen Keratosen zeigten 20 Patienten ein deutliches Ansprechen [4].

> Retinoide haben eine Normalisierungswirkung auf die Zelldifferenzierung, sind antiproliferativ und normalisieren die Apoptose der Zellen.

Schon aus diesem Grund haben sie ein Potenzial in der Krebsbehandlung [1, 2, $5,6,7]$.

Für HIV-bedingte Veränderungen besteht bereits eine Zulassung.

Bei früheren Rauchern betrachtet man den Verlust des Retinolsäurerezeptors $\beta$ (RAR- $\beta$ ) im Bronchialepithel als Biomarker einer beginnenden Neoplasie. In einer Untersuchung an 226 früheren Rauchern wurde placebokontrolliert entweder „9-cis-retinoic acid“ (RA; 100 mg), 13-cisRA (1 mg/kg) plus $\alpha$-Tocopherol oder Placebo gegeben. Nach 3 Monaten zeigten Bronchialbiopsien nur in der 9-cis-RAGruppe einen signifikanten Anstieg der RAR- $\beta$, Abnahme der Metaplasien und Anstieg des Serum- $\alpha$-Tocopherols $[12,17]$.

\section{Lymphome}

Positive Auswirkungen von $100 \mathrm{mg} / \mathrm{m}^{2}$ 9-cis-RA auf Non-Hodgkin-Lymphome und behandlungsrefraktäre T-Zell-Lymphome werden berichtet [39].

\section{Pagetoide Retikulose}

Über Alitretinoin bei diesem niedrigmalignen kutanen T-Zell-Lymphom (Sonderform der Mycosis fungoides) mit ungewöhnlich stark ausgeprägter Epidermotropie wird berichtet [32].

\section{Leukämie}

Alitretinoin spricht auf promyelozytische Leukämie (APL) an, nicht aber auf zervikale intraepitheliale Neoplasie $2 / 3$ (CIN 2/3) und auch nicht auf fortgeschrittene Tumoren unterschiedlichster Art, wenn diese unter Standardtherapie resistent waren [29].

In einem Fall koexistierender Psoriasis mit einem palmoplantaren kutanen TZell-Lymphom - eine seltene Kombination -, die unter hochpotenten Kortikosteroiden, Calcipotriol und SchmalbandUVB (NB-UVB) nicht gebessert werden konnte, erreichte Alitretinoin $30 \mathrm{mg}$ bereits nach 1 Monat deutliche Besserung, sodass im Anschluss eine 10-mg-Erhaltungstherapie durchgeführt werden konnte [7].

\section{Kombinationen}

Die Kombination von Tamoxifen und Alitretinoin ist gut verträglich und zeigte Antitumoraktivität bei metastatischem Brustkrebs. Als Phase-II-Dosierung wird angegeben $70 \mathrm{mg} / \mathrm{m}^{2} /$ Tag zusammen mit $20 \mathrm{mg} /$ Tag Tamoxifen [18].

Eine therapieresistente Mycosis fungoides vom Plaquetyp, die auf andere Therapie resistent war, konnte nach 6 Wochen unter 30 mg Alitretinoin in Kombination mit UVB $\left(1,2 \mathrm{~J} / \mathrm{cm}^{2}\right)$ fast vollständig zur Abheilung gebracht werden [24].

Ein 72-jähriger Patient, der seit 4 Jahren an Mycosis fungoides mit ausgeprägten Keratosen vor allem an den Füßen litt, zeigte kaum Verbesserung unter Acitretin und Calcineurininhibitoren über 1,5 Jahre, unter Alitretinoin jedoch nach 5 Monaten [23].

\section{Diskussion}

Viele der berichteten Off-label-Behandlungen sind Beobachtungen unter Einsatz von Alitretinoin bei Handekzemen, unter der sich auch andere Hautveränderungen besserten, bzw. nach Einsatz bei Dermatosen, die zunächst wie ein Handekzem imponierten.

Das Einsatzspektrum der Retinoide ist im Allgemeinen breit, wobei sich Alitretinoin durch seine antiinflammatorische und immunmodulatorische Wirkung unterscheidet und durch den Einfluss auf beide Retinoidrezeptoren der RAR- und RXR-Subtypen. Darüber hinaus könnten Einflüsse auf immunologische und endokrinologische Prozesse in der Haut bestehen. Im Gegensatz zu den bekannten Retinoiden Acitretin oder Isotretinoin zeigt Alitretinoin eine deutlich geringere haut- und schleimhautaustrocknende Wirkung.
Hautarzt 2013 · 64:748-751

DOI 10.1007/s00105-013-2593-2

C) Springer-Verlag Berlin Heidelberg 2013

\section{K. Fritz · G.S. Tiplica · C. Salavastru . M. Onder Alitretinoin und Off-label-Use}

\section{Zusammenfassung}

Orales Alitretinoin (9-cis-Retinsäure) ist ein körpereigenes Retinoid, das mit Vitamin A verwandt ist. Studien haben gezeigt, dass orales Alitretinoin wirksam und auch gut verträglich in der Behandlung des schweren chronischen Handekzems ist und für diese Indikation auch die Zulassung hat. Diese Übersichtsarbeit fasst aktuelle Studienergebnisse und Erfahrungsberichte zu anderen Indikationen („off-label“) mit Alitretinoin zusammen.

\section{Schlüsselwörter}

Vitamin-A-Säure-Rezeptoren - Retinoid .

Handekzem · Vitamin-A-Derivat .

Nebenwirkungen

\section{Off-label use of alitretinoin}

\section{Abstract}

Oral alitretinoin (9-cis retinoic acid) is an endogenous retinoid related to vitamin A. Studies have shown that oral alitretinoin is effective and well-tolerated in the treatment of severe chronic hand eczema, so that it is approved for this indication. This review summarizes new studies and clinical experience on the off-label use of alitretinoin.

\section{Keywords}

Vitamin A acid receptors - Retinoid · Hand eczema $\cdot$ Vitamin A derivative $\cdot$ Side effects

\section{》) Alitretinoin zeichnet sich durch seine antiinflammatorische und immunmodulatorische Wirkung aus}

Hauterkrankungen, bei denen eine gesteigerte Proliferation verbunden mit entzündlichen Prozessen eine Rolle spielt, könnten potenzielle Einsatzgebiete dieser Substanz darstellen, für die Einzelfallberichte vorliegen, aber noch keine Studie, die für andere Indikationen als dem Handekzem die Wirkung belegt.

Je mehr solche Berichte allerdings vorliegen, umso eher wird sich der Einsatz dieser noch relativ neuen Substanz auch „off label“ etablieren bzw. gar zu einer 
Nachzulassung und Zulassungserweiterung führen können.

Eingeschränkt wird ein breiterer Einsatz durch einige Retinoid-typische Begleiterscheinungen, die allerdings nur individuell auftreten, wie Leberbelastung, Erhöhung der Fettwerte, Kopfschmerzen, Sehstörungen und vor allem strikte Anwendung bei Frauen im gebärfähigen $\mathrm{Al}$ ter nur unter Kontrazeption.

\section{Fazit für die Praxis}

\section{- Alitretinoin hat eine antiinflamma- torische und immunmodulatorische Wirkung und beeinflusst die beiden Retinoidrezeptoren der RAR- und RXR-Subtypen. \\ - Hauterkrankungen, bei denen eine gesteigerte Proliferation verbunden mit entzündlichen Prozessen eine Rolle spielt, könnten potenzielle Ein- satzgebiete dieser Substanz darstel- len. \\ - Mögliche Indikationen sind insbeson- dere Störungen der Keratinisierung, der Hautfunktion und Hautbarriere und Einsatz bei malignen und präma- lignen Veränderungen, besonders Er- krankungen der weißen Zellreihe und Lymphome. Es sind weitere Berichte über Off-label-Behandlungen mit die- ser noch vergleichbar jungen Subs- tanz zu erwarten.}

\section{Korrespondenzadresse}

\section{Dr. K. Fritz}

Haut und Laser Centrum - Landau

Reduitstr. 13, 76829 Landau

drklausfritz@t-online.de

\section{Einhaltung ethischer Richtlinien}

Interessenkonflikt. K. Fritz, G.S. Tiplicab, C. Salavastru und M. Onder geben an, dass kein Interessenkonflikt besteht.

Dieser Beitrag beinhaltet keine Studien an Menschen oder Tieren.

\section{Literatur}

1. Abu J, Batuwangala M, Herbert K $K$, Symonds P (2005) Retinoic acid and retinoid receptors: potential chemo preventive and therapeutic role in cervical cancer. Lancet Oncol 6(9):712-720
2. Anolik JH, Giovanna JJD, Gaspari AA (1998) Effect of isotretinoin therapy on natural killer cell activity in patients with xeroderma pigmentosum. Br J Dermatol 138(2):236-241

3. Barnstedt SE (2012) Erfolgreiche Therapie eines Morbus Darier mit Alitretinoin. Hautarzt 2:139-141

4. Baumann L, Vujevich J, Halem M et al (2005) Openlabel pilot study of alitretinoin gel $0.1 \%$ in the treatment of photoaging. Cutis 76(1):69-73

5. Carneiro RV, Sotto MN, Azavedo LS et al (2005) Acitretin and skin cancer in kidney transplanted patients. Clinical and histological evaluation and immunohistochemical analysis of lymphocytes, natural killer cells and Langerhans' cells in sun exposed and sun protected skin. Clin Transplant 19(1):115-121

6. Chen K, Craig JC, Shumack S (2005) Oral retinoids for the prevention of skin cancers in solid organ transplant recipients: a systematic review of randomized controlled trials. Br J Dermatol 152(3):518523

7. Degos L (1990) Differentiating agents in the treatment of leukemia. Leuk Res 14(8):717-719

8. Emmert B, Hallier E et al (2011) Response of recalcitrant lichen planus to alitretinoin in 3 patients. J Am Acad Dermatol 65(2):58-60

9. Fritz K (2011) Therapieerfolg trotz latent aktivierbarer Poliomyelitis - ein Fallbericht. Dtsch Dermatol 11:2-3

10. Gånemo A, Sommerlund M, Vahlquist A (2012) Oral Alitretinoin in congenital Ichthyosis: a pilot study shows variable effects and a risk of central hypothy roidism. Acta Derm Venereol 92:256-257

11. Grahovac M, Molin S, Prinz J et al (2010) Treatment of atopic eczema with oral alitretinoin. Br J Dermatol 162(1):217-218

12. Han JY, Liu DD, Lee JJ et al (2005) 9-cis-retinoic acid treatment increases serum concentrations of a-tocopherol in former smokers. Clin Cancer Res 11(6):2305-2311

13. Irla N, Navarini A, Yawalkar N (2012) Alitretinoin abrogates innate inflammation in palmoplantar pustular psoriasis. Br J Dermatol 167(5):1170-1174

14. Jungersted JM, Høgh JK, Hellgren LI et al (2010) Changes in skin barrier during treatment with systemic alitretinoin: focus on skin susceptibility and stratum corneum ceramides. Arch Dermatol Res 302(9):653-666

15. Kolesnik M, Gollnick H, Bonnekob B (2013) Complete remission of alopecia areata under treatment of chronic hand eczema with alitretinoin. Eur J Dermatol 23(1):110-111

16. Kuhn A, Patsinakidis N, Luger T (2012) Alitretinoin for cutaneous lupus erythematosus. J Am Acad Dermatol 67(3):123-126

17. Kurie JM, Lotan R, Lee JJ et al (2003) Treatment of former smokers with 9-cis-retinoic acid reverses loss of retinoic acid receptor- $\beta$ expression in the bronchial epithelium: results from a randomized placebo-controlled trial. J Natl Cancer Inst 95(3):206-214

18. Lawrence JA, Adamson PC, Caruso R et al (2001) Phase I clinical trial of alitretinoin and tamoxifen in breast cancer patients: toxicity, pharmacokinetic, and biomarker evaluations. J Clin Oncol 15(19):2754-2763

19. Letulé V, Herzinger T, Ruzicka T, Molin S (2013) Treatment of Darier disease with oral alitretinoin. Clin Exp Dermatol 38(5):523-525

20. Meyer V, Goerge T, Luger TA, Beissert S (2011) Successful treatment of palmoplantar hyperkeratotic psoriasis with a combination of etanercept and alitretinoin. J Clin Aesthet Dermatol 4(4):45-46

21. Molin S, Ruzicka T (2010) Treatment of refractory pityriasis rubra pilaris with oral alitretinoin: case report. Br J Dermatol 163:208-234
22. Molin S, Ruzicka T (2010) Oral alitretinoin in lichen planus: two case reports. Acta Derm Venerol 90:523-524

23. Molin S, Ruzicka T (2009) Possible benefit of oral alitretinoin in T-Lymphoproliferative disease: a report of two patients with palmoplantar hyperkeratoticrhagadiform skin changes and mycosis fungoides or Sezary syndrome. Br J Dermatol 161(6):1420-1422

24. Motschenbacher S, Buder K, Goebler M, Kerstan A (2012) Combined oral alitretinoin and narrowband UVB treatment of cutaneous T cell lymphoma: favourable response after multiple ineffective systemic therapies. Eur J Dermatol 22(6):803-804

25. Noji S, Yamaai T, Koyama E et al (1989) Expression of retinoic acid receptor genes in keratinizing front of skin. FEBS Lett 259(1):86-90

26. Özkan F, Altmeyer P (2009) Alitretinoin als Therapieoption bei Patienten mit einer beruflich provozierten Psoriasis Palmaris. Dermatol Beruf Umwelt 57(3):119

27. Pinter A, Pätzold S, Kaufmann R (2011) Lichen planus of nails - successful treatment with Alitretinoin. J Dtsch Dermatol Ges 9(12):1033-1034

28. Petersen B, Jemec GB (2009) Alitretinoin - its use in intractable hand eczema and other potential indications. Drug Des Devel Ther 21(3):51-57

29. Rizvi NA, Marshall JL, Ness E et al (1998) Phase I study of 9-cis-retinoic acid (ALRT1057) in adults with advanced cancer. Clin Cancer Res 4(6):1437-1442

30. Sárdy M, Ruzicka T (2013) Successful therapy of refractory Hailey-Hailey disease with oral alitretinoin. Br J Dermatol. doi: 10.1111/bjd.12582 [Epub ahead of print]

31. Schmitt S, Inhoff O, Dippel E (2011) Oral alitretinoin for the treatment of recalcitrant pityriasis rubra pilaris. Dermatol 18(3):85-88

32. Schmitz L, BierhoffE, Dirschka T (2013) Alitretinoin: an effective treatment option for pagetoid reticulosis. J Dtsch Dermatol Ges. doi: 10.1111/ddg.12166 [Epub ahead of print]

33. Schopf M (2011) Lichen planus secondary to Hepatits $B$ vaccination, Successful treatment by alitretinoin (Poster EADV)

34. Srown T, Maher T, Astani R (2012) A Case of coexistent palmar/plantar cutaneous T-cell lymphoma and psoriasis treated with alitretinoin. (Poster AAD)

35. Tejera-Vaquerizo A et al (2012) Successsful treatment of recalcitrant chronic foot eczema. Actas Dermosifiliogr 103(2):931-932

36. Tosti A, Ricotti C, Romanelli P et al (2009) Evaluation of the efficacy of acitretin therapy for nail psoriasis. Arch Dermatol 145(3):269-271

37. Werchau S, Toberer F, Enk A, Helmbold P (2011) Keratitis-ichthyosis-deafness syndrome: response to alitretinoin and review of literature. Arch Dermatol 147(2):993-995

38. Wollenberg A (2011) Treatment of Dorfman-Chanarin syndrome with oral alitretinoin (Poster EADV)

39. Younes A, Cristofanilli M, Mclaughlin P et al (2000) Experience with 9-cis-retinoic acid in patients with relapsed and refractory non-Hodgkin's lymphoma. Leuk Lymphoma 40(1-2):79-85

40. Zamiri M, Munro C (2013) Successful treatment with oral alitretinoin in women of child-bearing potential with Darier's disease. Br J Dermatol. doi: 10.1111/ bjd.12348 [Epub ahead of print]

41. Zouboulis CC (2001) Retinoids-which dermatological indications will benefit in the near future? Skin Pharmacol Appl Skin Physiol 14(5):303-315 